\title{
Galaxies in the M81 Group and IC342/Maffei Complex:
} The Structure and Stellar Populations

\author{
Igor Drozdovsky \\ Astronomical Institute, St. Petersburg University, Russia \\ dio@astro.spbu.ru \\ Tikhonov Nikolay \\ Special Astrophysical Observatory, Russia Academy of Sciences, \\ ntik@sao.ru
}

\begin{abstract}
.
We present optical $V, R$, and $I$-band observations on the structure and stellar populations of the M81 Group and IC342/Maffei Complex galaxies. The images have been obtained with the $6 \mathrm{~m} \mathrm{BTA}$ and $2.5 \mathrm{~m}$ Nordic optical telescopes. Stellar photometry of several galaxies in these groups have been performed. The resulting colour-magnitude diagrams reveal the morphology of different stellar populations in these systems. Distances to the galaxies have been estimated from the tip of the red giant branch (TRGB), or from the brightest blue and red stars. For some galaxies the reddening, metallicity and surface profile parameters have been measured. We describe in more detail the results for four galaxies: IC 2574, Holmberg I, DDO 53 and IC 342. A more comprehensive project is underway.
\end{abstract}

\section{Introduction}

The M81 Group and the IC342/Maffei Complex are among of the nearest groups of galaxies, but their properties differ from the Local Group and from each other. The Local Group is a double system with the Milky Way and Andromeda subgroups. The M81 Group has one gravitationally dominant center, and in the IC342/Maffei complex there are several dominant galaxies. They have therefore provided a different environment for the evolution of their member galaxies. In particular gravitational interaction could influence the star-formation process and, possibly, galaxy morphology.

The M81 group of galaxies contains one large spiral (M81), two peculiar galaxies (M82 and NGC 3077), a few small spirals and a large number of dwarf galaxies. Further, there is evidence that a number of substantial interactions have occurred in this group in the recent past (Kennicutt et al. 1987, Yun, Ho, \& Lo 1994, Caldwell et al. 1998).

In our survey we also include NGC2403 companions located in the neighbourhood of the M81 Group as a possible subgroup. Their distances have been, up to now, a matter of debate. 
In the IC342/Maffei complex there are four massive galaxies (IC342, Maffei 1, Maffei 2, and Dwingeloo 1), one peculiar galaxy (NGC1569), two small spirals (UGCA105, and NGC1560), and several dwarfs.

While the IC342/Maffei complex is at low galactic latitude (mean extinction is about of two magnitudes in visual wavelengths $-A_{\mathrm{V}} \simeq 2 \mathrm{mag}$ ), the M81 Group has a small interstellar absorption $\left(A_{V} \simeq 0^{\mathrm{m}} 2\right)$. Despite its closer distance, (average IC342/Maffei complex distance modulus is about $26^{\mathrm{m}} 6$ versus $27^{\mathrm{m}} 8$ for the M81 Group) the member completeness of the IC342/Maffei Group is less than that of the M81 Group.

To study the resolved stellar populations of these galaxies, a multi-parameter problem needs to be solved - ages, metallicities, population fractions, and spatial variations, all of which depend crucially on knowledge of reddening and distance. All this problems can be solved by detailed multicolour CCD photometry.

During the last decade we have carried out a CCD survey of these groups to search for galaxies resolved into stars. This work was undertaken as a part of large survey of the Local Volume galaxies conducted by Dr. Karachentsev team at the Special Astrophysical Observatory. The CCD $V, R$ and $I$-band observations were carried out at the 6-meter telescope of Special Astrophysical Observatory, and the 2.5-meter Nordic Optical Telescope.

We combined two modes of the observations: a short exposure time widefield survey of the galaxian stellar populations (in bad seeing conditions) and deep observations of one or two fields in the galaxy periphery (in good seeing conditions). With good seeing we may expect to reach the TRGB in the $V$, $R$, and $I$ bands for the M81 Group, and the same in $\mathrm{R}$ and I bands for some of the galaxies of the IC342/Maffei complex. Our calculations showed that giant branch tip stars in galaxies at the distance of M81 should be resolved at apparent I-band magnitude of $m_{\mathrm{I}}^{\mathrm{TRGB}} \simeq 23.7$, and at the distance of IC342 at $m_{\mathrm{I}}^{\text {TRGB }} \simeq 23^{\mathrm{m}} 6$.

Table 1. Mean photometric data for the IC342/Maffei complex and M81 Group

\begin{tabular}{|c|c|}
\hline IC342/Maffei Complex & $\overline{M 81 \text { Group }}$ \\
\hline$\langle m-M\rangle=26^{\mathrm{m}} 63$ & $\langle m-M\rangle=27^{\mathrm{m}} 8$ \\
$\langle E(\mathrm{~B}-\mathrm{V})\rangle=00^{\mathrm{m}} 6$ & $\langle E(\mathrm{~B}-\mathrm{V})\rangle=0^{\mathrm{m}} 05$ \\
\hline \hline$A_{\mathrm{B}}=2^{\mathrm{m}} 55$ & $A_{\mathrm{B}}=0^{\mathrm{m}} 21$ \\
$A_{\mathrm{V}}=1^{\mathrm{m}} 95 m_{\mathrm{V}}^{\mathrm{TRGB}}=26^{\mathrm{m}} 8$ & $A_{\mathrm{V}}=0 .{ }^{\mathrm{m}} 16 m_{\mathrm{V}}^{\mathrm{TRGB}}=25^{\mathrm{m}} 6$ \\
$A_{\mathrm{R}}=1^{\mathrm{m}} 65 m_{\mathrm{R}}^{\mathrm{TRGB}}=25^{\mathrm{m}} 0$ & $A_{\mathrm{R}}=0.0^{\mathrm{m}} 14 m_{\mathrm{R}}^{\mathrm{TRGB}}=24^{\mathrm{m}} 6$ \\
$A_{\mathrm{I}}=1^{\mathrm{m}} 21 m_{\mathrm{I}}^{\mathrm{TRGB}}=23^{\mathrm{m}} 6$ & $A_{\mathrm{I}}=0^{\mathrm{m}} 10 m_{\mathrm{I}}^{\mathrm{TRGB}}=23^{\mathrm{m}} 7$ \\
\hline
\end{tabular}

\section{Results}

So far we have an optical database for most of the galaxies from these groups. This data base includes images and variable quality stellar photometry of the galaxies (Karachentsev et al. 1997). Here we describe in more detail the results 
for four galaxies: IC2574, Holmberg I and DDO53, and IC342. For some of the galaxies we been able to significantly improve distance estimations, and for others we will concentrate on stellar population studies.

\subsection{IC2574}

This object is a popular target for radio and X-ray observations, due to its bursting star-formation: a prominent supergiant shell has been detected in neutral hydrogen ( $\mathrm{HI}$ ) observations obtained with the Very Large Array. Massive star forming regions, as traced by $\mathrm{H} \alpha$ emission, are situated predominantly on the rim of this $\mathrm{HI}$ shell. This supports the view that the accumulated $\mathrm{H}$ I on the rim has reached densities which are high enough for secondary star formation to commence (Walter \& Brinks 1999).

Seven fields inside IC 2574 have been observed during 1998, and 1999 runs. After comparing the colour-magnitude diagrams from different fields we confirm the existence of the youngest population in this periphery region, and were found two chains of the active star formation regions.

\subsection{Holmberg I and DDO 53}

Holmberg I and DDO 53 are dwarf galaxies currently in a quiescent phase of star formation, the most numerous type in the M81 Group. The previous estimations of their distances were obtained by photometry of the brightest stars. Our CMDs show the upper $1^{\mathrm{m}} 5$ of the red giant branch. The $I$-band magnitudes of the red giant branch tip in both galaxies yield distances that are consistent with membership in the M81 Group. The mean color of the giant branch is used to establish the mean abundance of each galaxy. DDO 53, the more luminous galaxy, is significantly more metal-rich than Holmberg I. A young main sequence stellar component is present and clustered in centrally located clump, while older stars form more extended disk or halo. A small number of extended asymptotic giant branch stars are found beyond the RGB tip.

Table 2. Distance and metallicity estimates for Holmberg I and DDO 53

\begin{tabular}{|l|l|c|c|}
\hline Name & $m-M$ & $\mathrm{D}$ & {$[\mathrm{Fe} / \mathrm{H}]$} \\
\hline \hline HoI & $26^{\mathrm{m}} 69$ & $2.18 \mathrm{Mpc}$ & -2.0 \\
\hline DDO53 & $26^{\mathrm{m}} 95$ & $2.45 \mathrm{Mpc}$ & -1.2 \\
\hline
\end{tabular}

\subsection{IC342}

IC 342 is one of the nearest spiral galaxies with enhanced star formation. The intrinsic luminosity and distance to this galaxy (from 1.5 to $4.5 \mathrm{Mpc}$ ) have been a matter of some debate largely due to the uncertainty in the extinction estimates. We have estimated an average extinction and its variation from the position of the blue plume in the CMDs from the 10 fields.

We have found that the extremely remote region KK35 is at the same distance as IC342 $\left(M-m \simeq 26^{\mathrm{m}} 63 \pm 0^{\mathrm{m}} 35\right)$ through photometry of the brightest stars. This clump could either be a close satellite, or a remote scrap of the IC342 
spiral arm. At the moment a Hubble Space Telescope WFC image of KK35 is being analyzed (proposal 08192, program 5C3, "A Snapshot Survey of Probable Nearby Galaxies").

Despite high the galactic extinction, its central region is less obscured, due to almost face-on orientation of IC342, and can therefore be easier investigated than the center of the Milky Way (MW). This galaxy is in many aspects very similar to the MW.

\section{Conclusions}

The main results, some preliminary, are summarized as follows.

1. In two M81 Group galaxies the RGB was found and estimation of the distances on the basis of TRGB was made. For other galaxies of the IC342/Maffei complex and M81 Group distances were estimated by the brightest star method. This give the possibility to study the structure of these groups.

2. For some of the galaxies we estimated metallicity of the old disk stars based on position the RGB. Special effort was made to study the outer regions of galaxies. This data can be used in the galaxies primary stellar populations studies.

3. Interpretation of the observational CMDs by, e.g. superimposing isochrones to the data and deriving the ages of the stars populating the CMD, allowed us to confirm the idea of episodic (pulsation) star formation events in nearby galaxies. stars.

4. The time span between observations allowed us to search for variable

5. Population fractions (BSG, RSG, AGB, RGB) and spatial variations was determined for the M81 Group and IC342/Maffei complex galaxies. Modeling is in our future plans.

Acknowledgments. DI is grateful to organizers and sponsors of the Small Galaxy Groups Colloquium for travel support. This work was partly supported by RFBR Grant 97-02-17163.

\section{References}

Caldwell, N., Armandroff, T. E., Da Costa, G. S., Seitzer P., 1998, AJ, 115, 535 Karachentsev, I. D., Drozdovsky, I. O., Valtonen M., Takalo L., Heinämäki, \& Kajsin S., 1997, A\&AS, 124, 559

Kennicutt, R. C., Jr., Keel, W. C., van der Hulst, J. M., Hummel, E., \& Roettiger, K. A. 1987, AJ, 93, 1011

Walter, F., \& Brinks, E., 1999, AJ, 118, 273

Yun, M. S., Ho, P. T. P., \& Lo, K. Y. 1994, Nature, 372, 530 